\title{
MULHERES VÍTIMAS DE ABUSO SEXUAL EM UM MUNICÍPIO DA AMAZÔNIA
}

Women of sexual abuse in a amazonian municipality

Víctimas de abuso sexual en un municipio amazonico

Rosa Amélia Dias Batista Neta • Centro Universitário da Amazônia (UNAMA) E-mail: rosaneta.dias@hotmail.com

Silvane Oliveira Pimentel Guimarães • Centro Universitário da Amazônia (UNAMA) • E-mail: silvanepimentel2@gmail.com

Maria Conceição Cavalcante Farias • Enfermeira • Centro Universitário da Amazônia (UNAMA) • Mestrado em Bioengenharia pela Universidade Camilo Castelo Branco • E-mail: concefarias@yahoo.com.br

Luana Almeida dos Santos • Enfermeira • Especialista em Saúde da Família e Comunidade (UFOPA) • E-mail: luanah.orix@gmail.com

\section{Autora responsável pela correspondência:}

Luana Almeida dos Santos • E-mail: luanah.orix@gmail.com 


\section{RESUMO}

Introdução: A violência é um fenômeno presente no contexto histórico-social desde os primórdios dos tempos. Especial atenção vem merecendo a violência sexual (VS) contra a mulher. Objetivo: caracterizar quanto o perfil epidemiológico e sociodemográfico as mulheres vítimas de abuso sexual Metodologia: Este foi um estudo de campo, com abordagem descritiva e quantitativa. Os dados foram coletados do sistema operacional da Delegacia Especializada no Atendimento à Mulher, incluindo vítimas que sofreram violência sexual em Santarém, no estado do Pará nos anos de 2015 a 2018. Resultados: Foram encontradas 55 fichas de ocorrências, que corresponderam a 100\% da pesquisa: dez registros em 2015 (18\%), dezoito em 2016 (33\%), treze em 2017 (24\%), e quatorze em 2018 (25\%). Conclusão: O estudo teve objetivo alcançado, acrescentando o conhecimento dos profissionais da saúde a este assunto, em especial do enfermeiro. Como demonstrado, os dados estatísticos contribuem para tomada de medidas preventivas e de promoção contra o abuso sexual, salientando que o abuso sexual contra a mulher ainda é um tema a ser discutido.

Palavras-chave: Enfermagem; Saúde Pública; Violência Sexual.

\section{ABSTRACT}

Introduction: Violence has been a phenomenon present in the historical-social context since the dawn of time. Special attention has been given to sexual violence (VS) against women. Objective: to characterize the epidemiological and sociodemographic profile of women victims of sexual abuse. Methodology: This was a field study, with a descriptive and quantitative approach. Data were collected from the operational system of the Specialized Police Station for Women, including victims who suffered sexual violence in Santarém, in the state of Pará years 2015 to 2018. Results: 55 occurrence files were found, which corresponded to $100 \%$ of the survey: ten records in 2015 (18\%), eighteen in 2016 (33\%), thirteen in 2017 (24\%), and fourteen in 2018 (25\%). Conclusion: The study had an objective achieved, adding the knowledge of health professionals to this subject, especially nurses. As demonstrated, the statistical data contribute to preventive and promotional measures against sexual abuse, emphasizing that sexual abuse against women is still a topic to be discussed.

eywords: Nursing; Public health; Sexual Violence.

\section{SUMEN}

roducción: la violencia ha sido un fenómeno presente en el contexto histórico-social sde los albores del tiempo. Se ha prestado especial atención a la violencia sexual contra las mujeres. Objetivo: caracterizar el perfil epidemiológico y 
sociodemográfico de las mujeres víctimas de abuso sexual. Metodología: Este fue un estudio de campo, con un enfoque descriptivo y cuantitativo. Se recopilaron datos del sistema operativo de la Estación de Policía Especializada para Mujeres, incluidas las víctimas que sufrieron violencia sexual en Santarém, en el estado de Pará en los años 2015 a 2018. Resultados: se encontraron 55 archivos de casos, que correspondían al 100\% de la encuesta: diez registros en 2015 (18\%), dieciocho en 2016 (33\%), trece en 2017 (24\%) y catorce en 2018 (25\%). Conclusión: El estudio tuvo un objetivo logrado, agregando el conocimiento de los profesionales de la salud a este tema, especialmente las enfermeras. Como se demostró, los datos estadísticos contribuyen a las medidas preventivas y promocionales contra el abuso sexual, enfatizando que el abuso sexual contra las mujeres todavía es un tema a discutir.

Palabras clave: Enfermería; Salud pública; Violencia Sexual 


\section{ciênciála
pural}

\section{Introdução}

A violência é um fenômeno presente no contexto histórico-social desde os primórdios dos tempos. Especial atenção vem merecendo a Violência Sexual (VS) contra a mulher, que ocorre em qualquer espaço e comete qualquer pessoa. Sendo um grave problema contemporâneo de saúde pública mundial, tendo destaque em locais com condições socioeconômicas desfavoráveis ${ }^{1}$.

A violência sexual se apresenta de diversas formas, nas quais: aliciamentos, prática do ato sexual indesejável, exploração sexual e tráfico sexual. As vítimas que sofrem de violência sexual apresentam mudanças de comportamento, tais como: baixa autoestima, isolamento, depressão e constrangimento para dialogar sobre o ato violento que expõe sua intimidade ${ }^{2,3}$.

De acordo com as informações da atenção de integração à saúde, o atendimento à mulher em situação desse tipo de violência, deve apresentar incorporação de conhecimento com base científica, epidemiológicas sustentáveis e tecnológica, respeitando as peculiaridades de cada caso e de forma legalizada. Sendo de responsabilidade do profissional de saúde garantir à práxis assistencial ao direito constitucional $^{4}$.

Transcende fronteiras territoriais, educacionais, socioculturais e geracionais, configurando-se como um problema de magnitude mundial ${ }^{5}$. Mostra-se como uma das expressões da violência de gênero e firma-se como violação dos direitos humanos, sendo tema de preocupação mundial em virtude dos danos causados à saúde individual e coletiva. Vulnerabilidade física, emocional e social, que vão do abuso verbal ao estupro, e inclui diferentes tipos de coerção, como pressão social, intimidação e força física 6,7 .

No Brasil, uma, em cada dez mulheres com idade de 15 a 65 anos, tem uma ocorrência de violência sexual na vida. A cada 11 minutos uma mulher é estuprada no Brasil, segundo dados do Fórum Brasileiro de Segurança Pública (FBSP), divulgados n $2015^{8}$.

No Pará, a violência sexual é o segundo tipo de violência contra mulher mais rrado pela mídia, perdendo apenas para violência física ${ }^{9}$. Neste sentido, nos 
afirmam que a violência contra mulheres é um crime que atinge mulheres de qualquer faixa etária, classe social e etnia; muitos agressores são conhecidos das vítimas e em alguns casos, fazem parte do ciclo familiar ${ }^{10}$.

É Importante destacar que com a Lei $n^{\circ}$ 11.340/2006, mais conhecida como Lei Maria da Penha, é a lei que assegura o atendimento às mulheres em situação de violência e cria mecanismos de proteção às mulheres, bem como, o enfrentamento à impunidade da violência contra mulher, inclusive a sexual, sendo que a notificação imediata é fundamental para organização dos serviços a fim de que seja garantido o acesso às medidas de prevenção dos agravos resultantes da violência sexual em tempo oportuno ${ }^{4,11}$.

As mulheres que passaram por atos de violência se encontram suscetíveis no que tange o aparecimento de sintomas psiquiátricos, emocionais, tentativas de suicídio, uso de substâncias psicoativas, propensão de contrair infecção sexualmente transmissível (IST) e gravidez indesejada².

As consequências desses processos podem ser imediatas ou tardias, nas quais incluem ainda: aborto, complicações ginecológicas, doenças como Síndrome da Imunodeficiência Adquirida (HIV/AIDS) depressão, Transtorno de Estresse PósTraumático (TEPT) ${ }^{12}$.

A Lei $n^{0} 12.845 / 2013$, dispõe sobre o atendimento obrigatório e integral de pessoas em situação de violência sexual, o Decreto $n^{\circ} 7.958 / 2013$, estabelece diretrizes para o atendimento às vítimas de violência sexual pelos profissionais de segurança pública e da rede de atendimento do Sistema Único de Saúde (SUS) ${ }^{13}$.

Relatos sobre casos de estupro incidem nos mais diversos ambientes, desde o temido beco escuro, até mesmo o grande número de incidências ocorridas dentro da própria residência, pois a mesma pensa estar segura ${ }^{12}$.

Segundo a Organização Mundial da Saúde 14 , as mulheres jovens têm mais risco de sofrer estupro que mulheres mais velhas e mulheres que relatam baixos níveis de instrução (fundamental ou nenhum) têm de 2 a 5 vezes mais riscos de sofrerem iolência praticada pelo parceiro íntimo, que mulheres com alto nível de colaridade, portanto, o risco de violência sexual está para mulheres jovens, com ixo status socioeconômico, baixo nível de escolaridade, solteiras, 
separadas/divorciadas, e que fazem uso de substâncias psicoativas (álcool e droga).

No âmbito do Sistema Único de Saúde (SUS), as instituições envolvidas na atenção às pessoas em situação de violência sexual, devem assegurar cada etapa do atendimento que for necessária e isso inclui medidas de prevenção. A estruturação da atenção integral à saúde das pessoas em situação de violência sexual em rede é um passo importante para assegurar o cuidado, promoção e prevenção a esse público ${ }^{11}$.

Ação do cuidar realizada pela enfermagem precisa estar em conformidades com a Normas Técnicas do Ministério da Saúde, consistindo no saber técnico, com suas ações estão voltadas para o tratamento das lesões, prevenções IST's, e no auxílio da prevenção da gravidez indesejada. Com isso a assistência da equipe de enfermagem a mulher vítima de violência sexual antecede a uma prescrição médica para que seja feita a administração de medicamentos no sentido de diminuir os riscos ${ }^{15}$.

Faz-se necessário ampliar os pontos de atenção e fortalecer os já existentes, além de qualificar os das diversas áreas de atendimento à mulher em situação de violência para o atendimento humanizado e de qualidade ${ }^{16}$.

A educação em saúde é um importante instrumento que deve ser usada no enfretamento da VS fornecendo ações que estejam voltadas para locais de maior incidência, visando minimizar os casos ocorridos por meio de promoção e prevenção desse agravo pois a VS tem sido uma das principais causas de comorbidade a saúde, física e mental e coloca em risco a vida de muitas mulheres ${ }^{11}$. Objetivou-se caracterizar o perfil epidemiológico e sociodemográfico das mulheres vítimas de abuso sexual em um município da Amazônia.

\section{Metodologia}

Trata-se de um estudo de campo, descritivo com abordagem quantitativa. A pesquisa foi realizada na Delegacia Especializada de Proteção a mulher, do Município de Santarém, no estado do Pará.Tal instituição visa assegurar o combate à olência contra as mulheres, e tem como objetivo o fortalecimento e a efetiva mplementação de atendimento policial especializado, para assegurar tranquilidade população feminina vítima de violência. Através das atividades de investigação, 
prevenção e repressão dos delitos praticados contra a mulher, essa delegacia auxilia as mulheres agredidas, seus autores e familiares, a encontrarem o caminho da não violência, através de trabalho preventivo, educativo e curativo efetuados pelos setores jurídico e psicossocial.

Os dados foram obtidos através do boletim de ocorrência registrado no sistema operacional da referida delegacia. A partir desses dados foi elaborado o instrumento de coleta de dados, um questionário fechado, impresso, aplicado e personalizado através de nome de flor para não identificação da participante, preservando com isso a confidencialidade das informações prestadas, além da assinatura do Termo de Compromisso para Utilização de Dados (TCUD).

A amostra foi constituída de 55 fichas (100\%), dos boletins de ocorrência das mulheres que sofreram violência sexual, no período compreendido entre os anos de 2015 a 2018. Como critério de inclusão, foram selecionados aqueles que registraram mulheres que sofreram abuso sexual, com idade de 18 a 80 anos no município mencionado nos anos escolhidos.

Foram excluídos da pesquisa os boletins de ocorrência das mulheres que sofreram outros tipos de violência que não a sexual, que tinham idade inferior a 18 anos e superior a 80 anos e com registros feitos em anos que não correspondem ao de interesse da pesquisa. A análise de dados foi feita pela estatística descritiva e representada por meio de gráficos e tabelas.

Este estudo atendeu a todos os preceitos da resolução 466/2012 do Conselho Nacional de Saúde, que trata de pesquisas envolvendo seres humanos. Inicialmente o projeto foi encaminhado a Delegacia de Especialização de Atendimento à Mulher (DEAM) solicitando autorização para o desenvolvimento da pesquisa. Posteriormente o projeto foi enviado ao Comitê de Ética e Pesquisa com Seres Humanos do Instituto Campinense de Ensino Superior - ICES - Centro Universitário da Amazônia UNAMA localizado na Avenida Alcindo Cacela, No .287 , Bloco D, $5^{\circ}$ andar, bairro Umarizal, Município de Belém, Pará, obtendo aprovação conforme arecer $n^{0} 3.683 .382$. 


\section{ciêncíncia
puural}

\section{Resultados e Discussão}

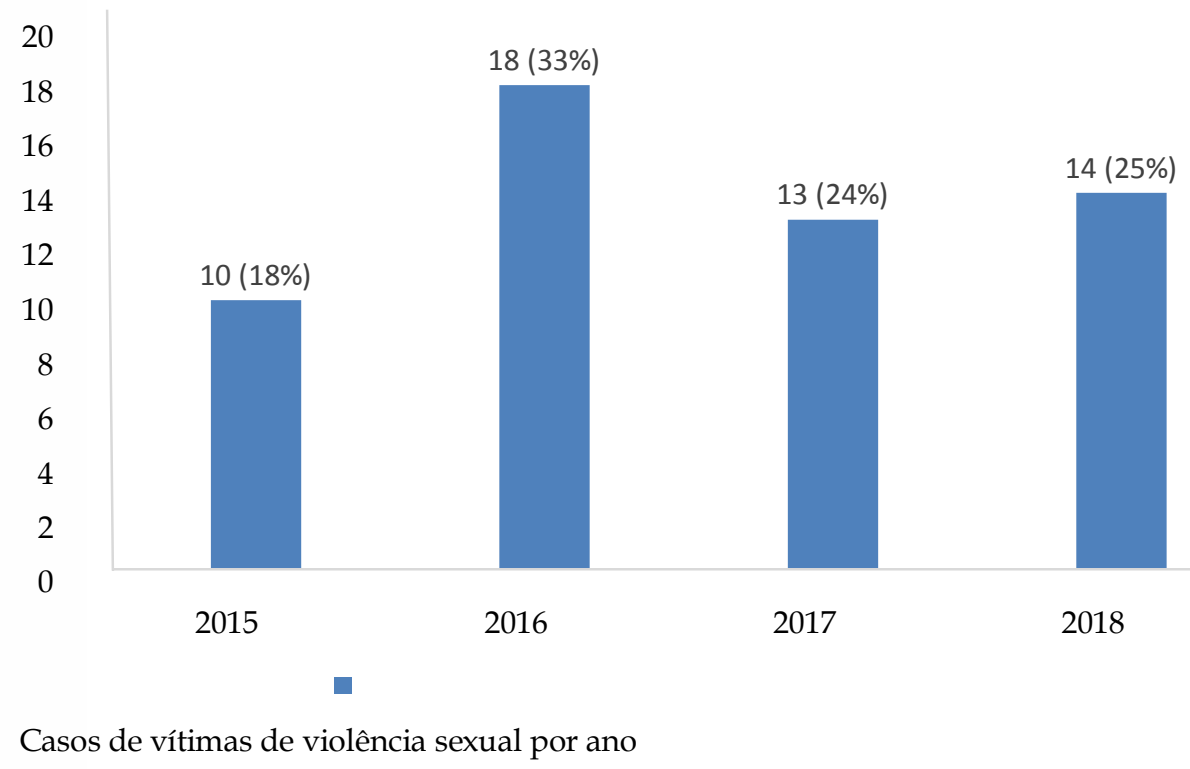

Gráfico 1- Porcentual de casos de violência sexual nos respectivas anos de 2015 a 2018. Santarém-PA, 2019.

Fonte: Boletins de ocorrências da DEAM no período de 2015-2018.

O gráfico (1) demonstra a distribuição do quantitativo de números de casos da Violência Sexual contra mulher por ano em Santarém. Observa-se que no ano de 2016 houve uma maior prevalência com 33\% de casos registrados, tendo uma pequena redução nos dois anos conseguintes 2017(24\%) e 2018(25\%).

Tabela 1- Dados epidemiológicos e Sociodemográficos de mulheres abusadas em 2015 a 2018. Santarém-PA, 2019.

\begin{tabular}{c|c|c}
\hline Características & $\mathrm{n}=55$ & $\%$ \\
\hline Zona & & 78,2 \\
\hline Urbana & 43 & 21,8 \\
\hline Rural & 12 & \\
\hline Faixa etária & & 72,7 \\
\hline $18-30$ anos & 40 & 12,7 \\
\hline $31-40$ anos & 7 & 12,7 \\
\hline $41-50$ anos & 7 & 1,8 \\
\hline $51-60$ anos & 1 & \\
\hline
\end{tabular}




\begin{tabular}{|c|c|c|}
\hline Raça & & \\
\hline Parda & 11 & 20 \\
\hline Não Identificado & 44 & 80 \\
\hline \multicolumn{3}{|l|}{ Escolaridade } \\
\hline Fundamental Incompleto & 11 & 20 \\
\hline Fundamental Completo & 3 & 5,4 \\
\hline Médio Incompleto & 15 & 27,3 \\
\hline Médio Completo & 12 & 21,8 \\
\hline Superior Incompleto & 5 & 9,1 \\
\hline Superior Completo & 2 & 3,6 \\
\hline Não Identificado & 7 & 12,7 \\
\hline \multicolumn{3}{|l|}{ Profissão } \\
\hline Estudante & 17 & 30,9 \\
\hline Doméstica & 9 & 16,4 \\
\hline Outros & 29 & 52,7 \\
\hline \multicolumn{3}{|l|}{ Estado Civil } \\
\hline Solteira & 41 & 74,5 \\
\hline União Estável & 9 & 16,4 \\
\hline Casada & 4 & 7,3 \\
\hline Outros & 1 & 1,8 \\
\hline
\end{tabular}

Fonte: Boletins de ocorrências da DEAM no período de 2015-2018.

Conforme a tabela 1, observou-se uma prevalência de quarenta casos em zona urbana que corresponde a $(78,2 \%)$ das vítimas. Corrobora com essa pesquisa da violência sexual em mulheres na cidade de Ribeirão Preto- SP de em que 86,6\% foram vitimadas no perímetro urbano ${ }^{17}$.

No que concerne faixa etária evidenciou-se que quarenta mulheres ou seja $(72,7 \%)$ das violentas tinham entre 18 e 30 anos. Dados semelhantes foram encontrados no estudo ${ }^{3}$, o qual mostrou que houve predomínio na faixa etária entre 18 a 27 anos. Vítimas de violência sexual são relativamente mais jovens que as sofrem com outros tipos de violências ${ }^{18}$.

Neste sentido o papel da humanização na saúde pode ser considerado um aminho para o cuidado integral, fortalecendo o vínculo entre as protagonistas uárias em um sistema de trocas recíprocas e positivas junto aos profissionais de 
saúde, podendo fazer circular uma espécie de solidariedade do cuidar e qualificação do cuidado ${ }^{19}$.

Na variável profissão, $17(30,9 \%)$ das vítimas alegaram como profissão estudante. Outras profissões 29 (52.7\%) foram citadas incluindo vendedora, balconista, aposentada, do lar, enfermeira, professora, diarista, secretária, cobradora, operadora de caixa, lavadeira, auxiliar de serviços gerais. Na pesquisa de Nunes et $\mathrm{al}^{2}$, foi constatado que $(50,9 \%)$ das agredidas eram estudantes, concordando assim com a presente pesquisa.

Sobre a caracterização da violência sexual em mulheres, na cidade de Ribeirão Preto-SP as mulheres solteiras foram as mais afetadas com predomínio de $(68,6 \%)$, dados que se mostram similares aos que foram encontrados nesse estudo posto que as mulheres solteiras foram também as mais atingidas $(74,5 \%)^{17}$.

Tabela 2- Características quanto aos casos de violência sexual em Santarém durante os anos 2015 a 2018. Santarém-PA, 2019.

\begin{tabular}{ccc}
\hline Características & $\mathrm{n}=55$ & $\%$ \\
\hline Vínculo & & 1,8 \\
\hline Pai & 1 & 7,3 \\
Marido e / ou companheiro & 4 & 36,4 \\
Desconhecido & 20 & 54,5 \\
Conhecidos & 30 & \\
\hline Denúncia & & 78,2 \\
\hline Vítima & 43 & 21,8 \\
Outros & 12 & 38,2 \\
\hline Local & & 43,6 \\
\hline Residência & 21 & 18,2 \\
\hline Via Pública & 24 &
\end{tabular}

Fonte: Boletins de ocorrências da DEAM no período de 2015-2018.

A presente pesquisa mostrou que a maioria das denúncias foi feita pelas róprias vítimas $(78,2 \%)$. Isso se deve ao fato da DEAM só aceitar, que outra pessoa nuncie, caso a vítima não possa responder por si, sendo responsável legal o 
denunciante, isso porque na maior parte dos casos em que outros fazem a denúncia a vítima não leva adiante, contribuindo assim com a impunidade do agressor.

Um estudo sobre violência sexual contra crianças e adolescentes ressalta que de modo geral, as pessoas têm receio de denunciar os casos que presenciam, principalmente quando se trata de violência sexual, pois os agressores podem rebelar-se contra seus denunciantes ${ }^{20}$.

Na variável local de ocorrência verificou-se que vinte e quatro dos casos de abuso sexual $(43,6 \%)$ ocorreu em via pública, seguido de vinte e um casos $(38,2 \%)$, na residência da vítima, dados reiterados pelo estudo de Oliveira ${ }^{16}$ que relata $65,21 \%$ de abusos ocorridos na residência e 11,26\% em via pública.

\section{Conclusão}

Os resultados mostraram que as notificações das vítimas de abuso sexual, geralmente são feitas pela própria vítima, que são encaminhadas para a delegacia especializada, em seguida é avaliada por toda a equipe multiprofissional, porém ainda existem muitos casos que não são notificados por medo de grandes represálias dos acusados.

As mulheres que sofrem abuso sexual padecem de vários tipos de lesões físicas, nas genitálias, psicológicas conforme mostra o sistema operacional. Contudo, há a necessidade de chamar a atenção dos governantes com relação às medidas de educação em saúde, nas mídias, usando a internet, emissoras, rádios para divulgação de informações. Para o enfermeiro, é importante o desenvolvimento das políticas sociais para enfrentamento do abuso sexual em mulheres, visando a diminuição desse ato que é cruel afetando profundamente as vidas dessas pessoas.

Dessa forma, a capacitação de profissionais qualificados para atuar nas unidades de saúde é necessária, tanto na promoção quanto no atendimento das vítimas.

A portaria ampara os profissionais da unidade básica para atuar no atendimento a vítima, mas o mesmo não possui a devida qualificação para atendê-la nem materiais necessários para acolher a mesma. O governo deveria investir mais 
na capacitação dos profissionais e na orientação dos usuários quanto aos atendimentos ofertados na rede de saúde.

\section{Referências}

1.Baptista RS, Chaves OBBM, França ISX, Sousa FS, Oliveira MG, Leite CCS. Violência sexual contra mulheres: a prática de enfermeiros. Universidade Estadual da Paraíba. Campina Grande, PB. Brasil. Revista Rene. Paraíba (PB)16 v.(2):p. 210- 217, 07 abr, 2015.

Disponível em: <http:/ / repositorio.ufc.br/bitstream/riufc/12654/1/2015_art_rsbaptista.pdf> Acesso em 11 de mar. 2019.

2. Nunes MCA, Lima RFF, Morais NA. Violência Sexual contra Mulheres: um Estudo Comparativo entre Vítimas Adolescentes e Adultas Psicologia Ciência e Profissão Out/Dez. 2017 v. 37 n4, 956-969 Universidade de Fortaleza, CE, Brasil. Disponível em <https:/ / doi.org/10.1590/1982-3703003312016> Acesso em 12 fev 2019.

3 Araújo RPD, Sousa FMS, Feitosa VC, Coêlho DMM, Sousa MFA . Perfil Sociodemográfico e Epidemiológico da Violência Sexual Contra as Mulheres em Teresina/Piauí. Brasil. REUFSM Revista de Enfermagem da UFSM2014 Out/Dez; 4. Teresina (PI). $\quad$ v.4 n.740.p. 2 11, 11 dez. 2014. Disponível em: <http://www/periodicos.ufsm.br/reufsm/article/view/14519/pdf> Acesso em: 02 de Fev. de 2019.

4. Brasil Secretaria de Atenção à Saúde Departamento de Ações Programáticas Estratégicas prevenção e tratamento dos agravos resultantes da violência sexual contra mulheres e adolescentes. Norma Técnica. $3^{\mathrm{a}}$ edição atualizada e ampliada $1^{\mathrm{a}}$ reimpressão. Série A. Normas e Manuais Técnicos. Série Direitos Sexuais e Direitos Reprodutivos - Caderno $\mathrm{n}^{\mathrm{o}}$ 6. Brasília - DF 2012. Disponível em: <http://bvsms.saude.gov.br/bvs/publicacoes/prevencao_agravo_violencia_sexual_ m ulheres_3ed.pdf> Acesso em 28 de out. de 2019.

5. Bezerra JF, Silva RM, Cavalcante LF, Nascimento JL, Vieira LJES, Moreira GAR. Conceitos, causas e repercussões da violência sexual contra a mulher na ótica de profissionais de saúde. Fortaleza Fortaleza-Ceará, Brasil. Revista Brasileira em Promoção da Saúde, Fortaleza (CE) v. 29, n. 1, 23 mar. 2016, p. 51-59,. Disponível em: <http:// www.redalyc.org/articulo.oa?id=40846964008> Acesso 8 fev. 2019.

6. Moreira RAG, Freitas MK, Cavalcanti FL, Vieira LJES, Silva RM . Qualificação de profissionais da saúde para a atenção às mulheres em situação de violência sexual de an. de 2016. Rio de Janeiro, v. 16 n. 3, p. 1.039-1.055, set./dez. 2018. Disponível em: http://www.scielo.br/pdf/tes/v16n3/1678-1007-tes-1981- 7746sol00156.pdf> cesso em 12 de mar.2019.

abral AMG. Buissa ACR, Carnier CC, Miyazaki ET, Gongora DVN, Lucânia ER, et Perfil de mulheres vítimas de abuso sexual atendidas em hospital de ensino Arq. 
Ciênc. Saúde. 2015 jul-set. Disponível em: <http://www.cienciasdasaude.famerp.br/index.php/racs/article/download/237/1 05/> Acesso em 21 março de 2019.

8. Delziovo CR, Coelho EBS, D'orsi E, Rubia LS. Violência sexual contra a mulher e o atendimento no setor saúde em Santa Catarina - Brasil. Ciência \& Saúde Coletiva, 23(5):1687-1696, 2018 Disponível em: <http://www.unifesp.br/reitoria/dci/ edicao- atualentreteses/item/2590-um-estupro-a-cada-11-minutos> Acesso em 21 out 2019.

9. Augusto AO, Azevedo LVL, Sena LX, Silva AF, Gomes VR, Santos ACB. Mapeamento dos casos de violência contra a mulher na região metropolitana de Belém narrados pela mídia impressa do estado do Pará. Brasil. Revista Paraense de Medicina.

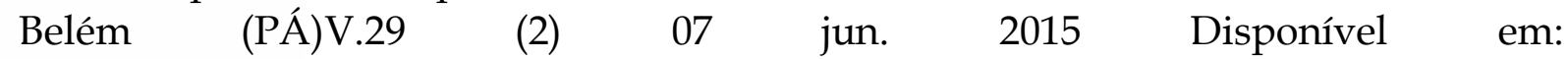
<http://bases.bireme.br/cgibi/wxislind.exe/iah/online/?IsisScrip> Acesso em out. de 2019.

10 Souto RQ, Leite C.C.S, França I.S.X. Cavalcanti A.L. violência sexual contra mulheres portadoras de necessidades especiais: perfil da vítima e do agressor. Cogitare Enferm. Jan/Mar2012 Disponível em < http:/ / > Acesso em 20 fev 2019.

11. Brasil. Ministério da justiça, secretaria de políticas para as mulheres/PR. Norma técnica atenção humanizada às pessoas em situação de violência sexual com registro de informações e coleta de vestígios. 2015, Tiragem: $1^{\mathrm{a}}$ edição - $1^{\mathrm{a}}$ impressão - 2015 5.000 exemplares, Brasília - DF 2015. Disponível em: <http://bvsms.saude.gov.br/bvs/publicacoes/atencao_humanizada_pessoas_viole nci a_sexual_norma_tecnica.pdf > Acesso em 28 de out. de 2019.

12. Sousa RF. Cultura do estupro: prática e incitação à violência sexual contra mulheres. Rev. Estud. Fem. [online]. 2017, vol.25, n.1, pp.9-29. ISSN 18069584. http://dx.doi.org/10.1590/1806-9584.2017v25n1p9. Disponível em: <http://www.scielo.br/pdf/ref/v25n1/1806-9584-ref-25-01-00009.pdf> Acesso em: 12 mar 2019.

13. Pinto SSL, Oliveira IMP, Pinto ESS, Leite CBC, Melo AN, Deus MCBR. Políticas públicas de proteção à mulher: avaliação do atendimento em saúde de vítimas de violência sexual. Ciência \& Saúde Coletiva, 22(5):1501-1508, 2016. Disponível em: <http://www.scielo.br/pdf/csc/v22n5/14138123-csc-22-05-1501.pdf> Acesso em: 02 de Fev. de 2019

14. Organização Mundial da Saúde OMS. Prevenção da violência sexual e da violência lo parceiro íntimo contra a mulher: ação e produção de evidência 1- prevenção rganização Mundial da Saúde, 2012 pág 30 a 31. Disponível em: https:/ /apps.who.int/iris/bitstream/handle/10665/44350/9789275716359_por.pdf sessionid $=44 F F 03422 \mathrm{D} 29 \mathrm{~F} 672453399201 \mathrm{~B} 98 \mathrm{~A} 72 \mathrm{E}$ ?sequence $=3>$ Acesso em: $30 \mathrm{de}$ ar. de 2019. 
15. Oliveira AFS, Emanuelle T, Barreto CA. O cuidar em enfermagem à mulher vítima de violência sexual Brasil. Revista Saúde em Foco - Edição no 11 - Ano: 2019 p. 567573. http://portal.unisepe.com.br/unifia/wpcontent/uploads/sites/10001/2019/05/051 _O -cuidar-da-Enfermagem.pdf Acesso em: 14 nov 2019

16. Oliveira CAB. Perfil da vítima e características da violência contra a mulher no estado de Rondônia - Brasil. Rev Cuid. 2017; Rondônia (RO) v.10 n. (1)573 : p.02. Disponível em: <http:/ / dx.doi.org/10.15649v10i1.573> Acesso em 30 de Mar. de 2019.

17 Souza ACS. Caracterização da Violência Sexual em Mulheres na cidade de Ribeirão Preto- SP, 2012 108p., dissertação de mestrado, apresentada à Faculdade de Medicina de Ribeirão Preto/USP. Disponível em <https://www.teses.usp.br > tde-11082015131209> Acesso em 06 out 2019.

18. Patricio JA, Violência contra as mulheres: processos e contextos de vitimização , Fórum Sociológico [Online], 25 | 2014, posto online no dia 10 novembro 2014, consultado o 19 abril 2019. URL: Disponível em: http://journals.openedition.org/sociologico/902; DOI: 10.4000/sociologico.902. Acesso em: 03 nov 2019.

19. Cunha ATR, Vilar RLA, Melo RHV, Silva AB, Rodrigues MP. Percepções de usuários sobre humanização na estratégia saúde da família: um estudo ancorado na teoria da dádiva. Revista Ciência Plural, v. 3, n. 3, p. 16-31, 22 abr. 2018.

20. Santana JSS, Santana RPS, Lopes ML. Violência sexual contra crianças e adolescentes: análise de notificações dos conselhos tutelares e departamento de polícia técnica Bahia Brasil. Revista Baiana de Saúde Pública Bahia (BA) v.35, supl.1, p.68-86 jan./jun.2011Disponível em:<http://files.bvs.br/upload/S/01000233/2011/ v.35, n. Sup 11/a2299.pdf> Acesso em: 14 nov 2019. 